\title{
Appendix: Aiming for Efficiency Rather than Proficiency
}

\author{
Derek Neal
}

June 12, 2010

University of Chicago and NBER 
Consider an education authority who is charged with educating $N$ students, indexed $n=1,2, \ldots N$. Let $s_{n}$ be a skill index that describes the skill level of student $n$ at the beginning of the current period and let $s_{n}^{\prime}$ denote the skill level of this same child at the end of the period. Assuming that no two students begin with the exact same baseline skill level, we can always order students according to their baseline skill levels, $\left(s_{1}<s_{2}<s_{3}<\ldots s_{N}\right)$.

For ease of expostion, assume that all education takes place through one on one tutoring and that the authority can hire tutors in a competive labor market at a wage rate of $w$ per hour. Further, assume that the authority possesses a monitoring technology that allows the authority to costlessly verify that tutors are providing effective instruction.

Next, define $e_{n}$ as the hours of effective tutoring devoted to child $n$, and assume the following skill production function, $\Delta s_{n}=s_{n}^{\prime}-s_{n}=f\left(e_{n}\right)$, which asserts that skill growth during the period for student $n$ is determined by the tutoring effort devoted to student $n$. Assume that the function $f($.$) is weakly$ increasing and strictly concave. These assumptions imply that extra tutoring is typically productive and never harmful, but because each student has a fixed amount of time and energy, the marginal skill gains that result from allocating additional tutoring to a given student diminsh as he receives more tutoring, and at extremely high tutoring levels, these marginal returns may become zero due to child fatigue.

Given this setting, define

$$
V=V\left(s_{1}^{\prime}, s_{2}^{\prime}, . . s_{N}^{\prime}\right)
$$

as society's educational objective function. The function ranks the possible vectors of child skill levels at the end of the period from society's perspective. Assume that $V$ is continuously increasing in all of its arguments and concave. These conditions imply that increasing the skill of any child is a good thing assuming that the skill levels of others are not harmed and further any skill improvement by a less skilled child is at least as valuable to society as a comparable improvement among a highly skilled child. ${ }^{1}$ Further, assume that student identity does not directly enter $V$, e.g. in the case where there are only two students, $V\left(s_{1}^{\prime}=3, s_{2}^{\prime}=5\right)$ provides the same value for society as $V\left(s_{1}^{\prime}=5, s_{2}^{\prime}=3\right)$.

Now, imagine that the job of an education authority is to maximize this educational objective function, $V$, by choosing the best allocation of tutoring time among students, $\left(e_{1}, e_{2}, . . e_{N}\right)$, subject to a budget constraint which limits the total spending on tutors, $B \geq \sum_{n=1}^{N} w e_{n}$. It is straightforward to demonstrate that the optimal allocation of tutoring efforts among students must satisfy the following condition:

$$
\frac{\partial V}{\partial s_{1}^{\prime}} \frac{\partial s_{1}^{\prime}}{\partial e_{1}}=\frac{\partial V}{\partial s_{2}^{\prime}} \frac{\partial s_{2}^{\prime}}{\partial e_{2}} \ldots=\frac{\partial V}{\partial s_{N}^{\prime}} \frac{\partial s_{N}^{\prime}}{\partial e_{N}}
$$

\footnotetext{
${ }^{1}$ It is important to note that I am not taking a stand on why society value student skill. $V$ may reflect many factors, e.g. skills make people more productive, skills make people better neighbors and citizens, etc.
} 
If this condition does not hold, then the authority should reallocate resources toward the students whose further tutoring generates the greatest social gains until the returns to tutoring are equalized at the margin over all students.

Given the assumptions made this far, accountabilty systems built around proficiency standards will not generate outcomes that satisfy this condition. Assume that, given a diverse set of baseline skill levels, $\left(s_{1}<s_{2}<s_{3}<\ldots s_{N}\right)$, the education authority allocates tutoring resources to make sure that all students achieve a common proficiency standard, so that a significant number of students achieve the same final skill level, $\bar{s}^{\prime}$, and the remaining students have even higher skill levels. This allocation of tutoring effort cannot satisy the optimality condition above and is therefore socially wasteful. Note that the value to society of allocating a small amount of extra tutoring to student $n$ is the product of the the rate at which $V$ increases as $s_{n}^{\prime}$ increases and the rate at which $s_{n}^{\prime}$ increases as student $n$ receives additional tutoring. Since student identity does not enter $V$, this first effect, $\frac{\partial V}{\partial s_{n}^{\prime}}$, is the same for all students who ended the period at a common proficiency level, $\bar{s}^{\prime}$. However, if the students, who end the year at a common proficiency level, began the year at different levels of baseline achievement, the second term, $\frac{\partial s_{n}^{\prime}}{\partial e_{n}}$, must be different among these students. The presence of diminishing returns implies that, under a policy that mandates universal proficiency, the marginal returns to tutoring are lowest among those who began the farthest below the proficiency standard. Further, if marginal returns to tutoring effort reach zero at some point, certain proficiency standards may not be feasible for some students.

The key assumption here is that there are diminishing returns from investing additional resources in any particular child. I trust that most readers and certainly all parents understand how natural the assumption of diminshing returns is in this context. Children have finite time and attention, and this fixed factor constrains the ability of educators to increase the skill of any student simply by devoting more attention to her. 